\title{
IMITATOR: a Tool for Synthesizing Constraints on Timing Bounds of Timed Automata ${ }^{\star}$
}

\author{
Étienne André \\ LSV - ENS de Cachan \& CNRS, France
}

\begin{abstract}
We present here ImITATOR, a tool for synthesizing constraints on timing bounds (seen as parameters) in the framework of timed automata. Unlike classical synthesis methods, we take advantage of a given reference valuation of the parameters for which the system is known to behave properly. Our aim is to generate a constraint such that, under any valuation satisfying this constraint, the system is guaranteed to behave, in terms of alternating sequences of locations and actions, as under the reference valuation. This is useful for safely relaxing some values of the reference valuation, and optimizing timing bounds of the system. We have successfully applied our tool to various examples of asynchronous circuits and protocols.
\end{abstract}

\section{Context}

Timed automata [1] are finite control automata equipped with clocks, which are real-valued variables which increase uniformly. This model is useful for reasoning about real-time systems, because one can specify quantitatively the interval of time during which the transitions can occur, using timing bounds. However, the behavior of a system is very sensitive to the values of these bounds, and it is rather difficult to find their correct values. It is therefore interesting to reason parametrically, by considering that these bounds are unknown constants, or parameters, and try to synthesize a constraint (i.e., a conjunction of linear inequalities) on these parameters which will guarantee a correct behavior of the system. Such automata are called parametric timed automata (PTA) $[2,11]$.

The synthesis of constraints for PTA has been mainly done by supposing given a set of "bad states" (see, e.g., [8,9]). The goal is to find a set of parameters for which the considered timed automaton does not reach any of these bad states. We call such a method a bad-state oriented method. By contrast, we present in this paper a tool based on a good-state oriented method.

\section{Principle of Imitator}

The tool Imitator (Inverse Method for Inferring Time AbstracT behaviOR) implements the algorithm InverseMethod, described in [4]. We assume given a

\footnotetext{
* This work is partially supported by the Agence Nationale de la Recherche, grant ANR-06-ARFU-005, and by Institut Farman (ENS Cachan).
} 
system modeled by a PTA $\mathcal{A}$. Whereas bad-state oriented methods consider a set of bad states, IMITATOR considers an initial tuple $\pi_{0}$ of values for the parameters, under which the system is known to behave properly. When the parameters are instantiated with $\pi_{0}$, the system is denoted by $\mathcal{A}\left[\pi_{0}\right]$. Under certain conditions, the algorithm InverseMethod generalizes this good behavior by computing a constraint $K_{0}$ which guarantees that, under any parameter valuation $\pi$ satisfying $K_{0}$, the system behaves in the same manner: the behaviors of the timed automata $\mathcal{A}[\pi]$ and $\mathcal{A}\left[\pi_{0}\right]$ are (time-abstract) equivalent, i.e., the traces of execution viewed as alternating sequences of locations (or "control states") and actions are identical. This is written $\mathcal{A}[\pi] \equiv_{T A} \mathcal{A}\left[\pi_{0}\right]$. More formally, the algorithm InverseMethod solves the following inverse problem [4] for acyclic systems (i.e., with only finite traces) by computing a constraint $K_{0}$ such that:

1. $\pi_{0}=K_{0}$,

2. $\mathcal{A}[\pi] \equiv_{T A} \mathcal{A}\left[\pi_{0}\right]$, for any $\pi \models K_{0}$.

A practical application is to optimize (either decrease or increase) the value of some element of $\pi_{0}$, as long as it still satisfies $K_{0}$. This is of particular interest in the framework of digital circuits, in order to safely minimize some stabilization timings (typically "setup" or "hold").

The tool Imitator is available on its Web page ${ }^{1}$.

\section{General Structure}

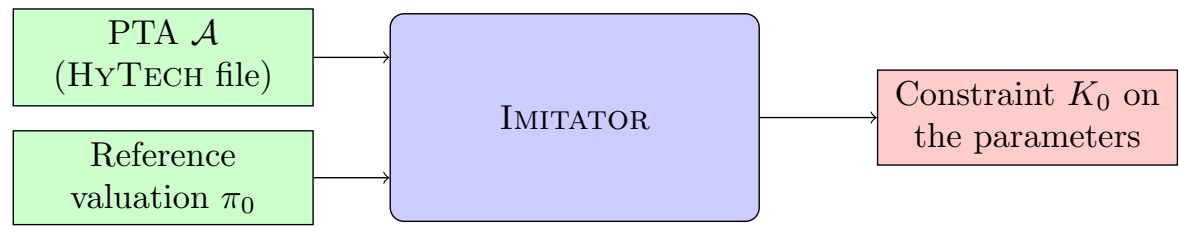

As depicted above, Imitator takes as inputs a PTA described in HyTeCH syntax, and a reference valuation $\pi_{0}$. The aim of the program is to output a constraint $K_{0}$ on the parameters solving the inverse problem.

The algorithm InverseMethod on which IMITATOR relies can be summarized as follows. Starting with $K:=$ True, we iteratively compute a growing set of reachable symbolic states. A symbolic state of the system is a couple $(q, C)$, where $q$ is a location of the PTA, and $C$ a constraint on the parameters ${ }^{2}$. When a $\pi_{0}$-incompatible state $(q, C)$ is encountered (i.e., when $\left.\pi_{0} \not \models C\right), K$ is refined as follows: a $\pi_{0}$-incompatible inequality $J$ (i.e., such that $\pi_{0} \not \models J$ ) is selected within $C$, and $\neg J$ is added to $K$. The procedure is then started again with this new $K$, and so on, until the whole set of reachable states $\left(\right.$ Post $\left.^{*}\right)$ is computed.

A simplified version of algorithm InverseMethod is given below, where the clock variables have been disregarded for the sake of simplicity. We denote by

\footnotetext{
${ }^{1}$ http://www.lsv.ens-cachan.fr/ andre/IMITATOR

${ }^{2}$ Strictly speaking, $C$ is a constraint on the clock variables and the parameters, but the clock variables are omitted here for the sake of simplicity. See [4] for more details.
} 
$\operatorname{Post}_{\mathcal{A}(K)}^{i}(S)$ the set of symbolic states reachable from $S$ in exactly $i$ steps of $\mathcal{A}(K)$, and $\exists X: C$ denotes the elimination of clock variables in constraint $C$.

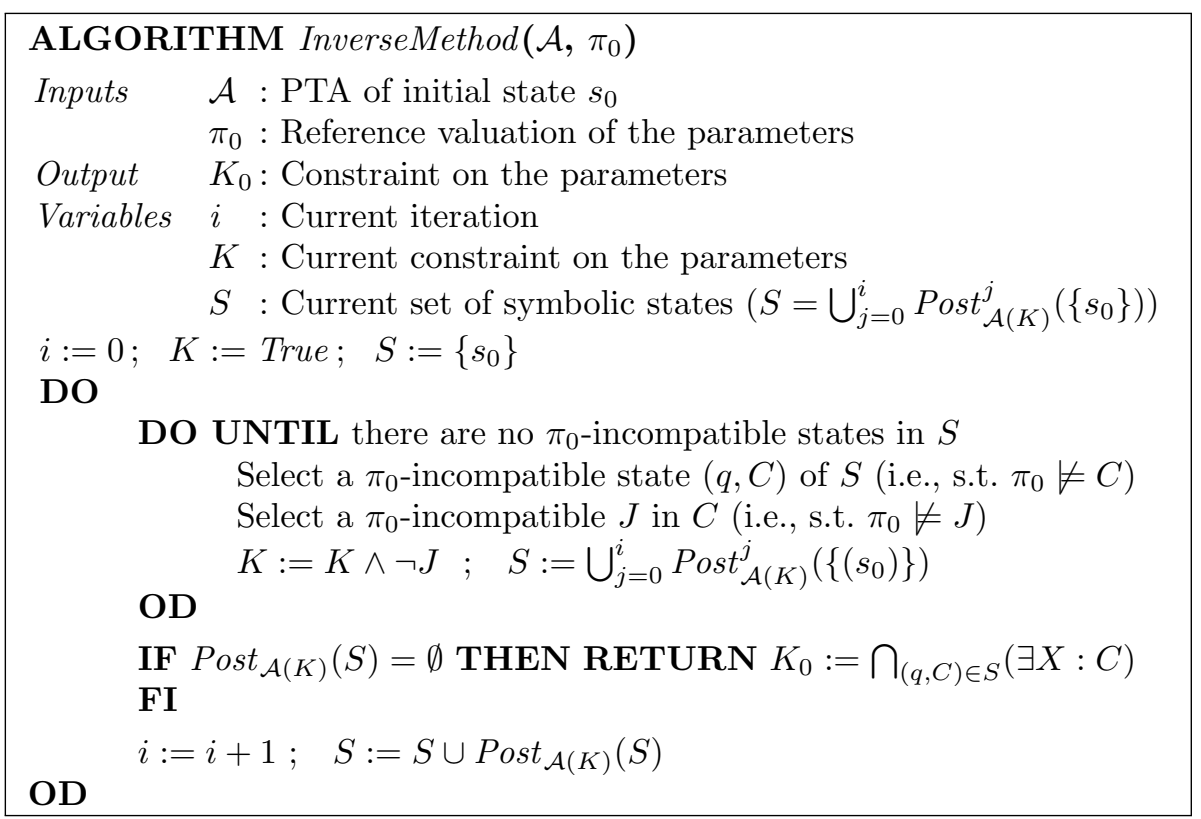

This algorithm terminates and solves the inverse problem for acyclic systems. The acyclic class is interesting for hardware verification, e.g., when analyzing synchronous circuits over a fixed number (typically, 1 or 2) of clock cycles.

Imitator is a program written in Python, that drives HyTech [10] for the computation of the Post operation. The Python program contains about 1500 lines of code, and it took about 4 man-months of work.

Remark. In order to handle cyclic examples, one modifies the algorithm by replacing, in the IF condition, $\operatorname{Post}_{\mathcal{A}(K)}(S)=\emptyset$ by $\operatorname{Post}_{\mathcal{A}(K)}(S) \subseteq S$. In that case, we ensure termination more often (see [4]). However, we do not guarantee any longer the identity of traces, but only the identity of reachable locations. This is interesting when $\mathcal{A}\left[\pi_{0}\right]$ is known to avoid a given bad location because, in this case, $\mathcal{A}[\pi]$ is also guaranteed to avoid this bad location, for any $\pi=K_{0}$.

\section{An Illustrating Example}

We consider an asynchronous "D flip-flop" circuit described in [7] and depicted on Fig. 1. It is composed of 4 gates $\left(G_{1}, G_{2}, G_{3}\right.$ and $\left.G_{4}\right)$ interconnected in a cyclic way, and an environment involving two input signals $D$ and $C K$. The global output signal is $Q$. Each gate $G_{i}$ has a delay in the parametric interval $\left[\delta_{i}^{-}, \delta_{i}^{+}\right]$, with $\delta_{i}^{-} \leq \delta_{i}^{+}$. There are 4 other parameters (viz., $T_{H I}, T_{L O}, T_{\text {setup }}$, and $\left.T_{\text {hold }}\right)$ used to model the environment. Each gate is modeled by a PTA, 

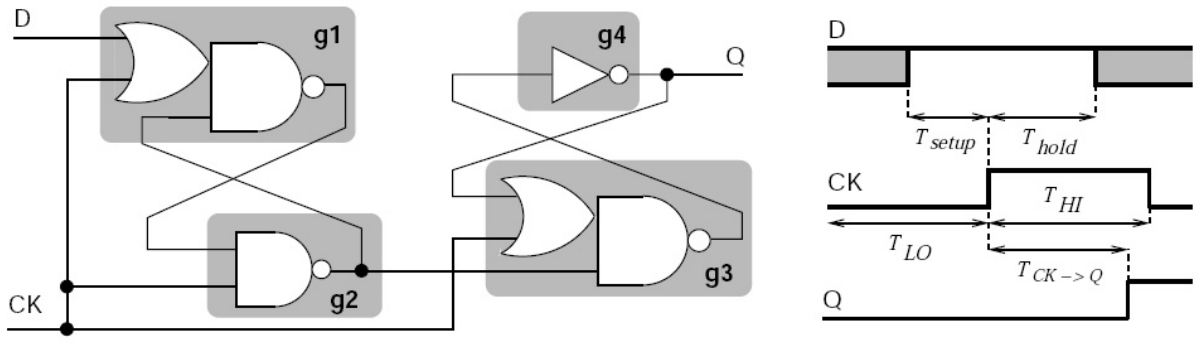

Fig. 1. Flip-flop circuit

as well as the environment. We consider an inertial model for gates, where any change of the input may lead to a change of the output (after some delay). The PTA $\mathcal{A}$ modeling the system results from the composition ${ }^{3}$ of those 5 PTAs. The output signal of a gate $G_{i}$ is named $g_{i}$ (note that $Q=g_{4}$ ). The rising (resp. falling) edge of signal $D$ is denoted by $D^{\uparrow}$ (resp. $D^{\downarrow}$ ) and similarly for signals $C K, Q, g_{1}, \ldots, g_{4}$. We consider the following instantiation $\pi_{0}$ of the parameters :

$$
\begin{aligned}
& T_{H I}=24 \quad T_{L O}=15 \quad T_{\text {setup }}=10 \quad T_{\text {hold }}=17 \quad \delta_{1}^{-}=7 \quad \delta_{1}^{+}=7
\end{aligned}
$$

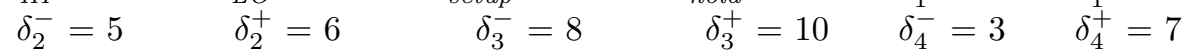

We consider an environment starting from $D=C K=Q=0$ and $g_{1}=$ $g_{2}=g_{3}=1$, with the following ordered sequence of actions for inputs $D$ and $C K: D^{\uparrow}, C K^{\uparrow}, D^{\downarrow}, C K^{\downarrow}$, as depicted on Fig. 1 right. Therefore, we have the implicit constraint $T_{\text {setup }} \leq T_{L O} \wedge T_{\text {hold }} \leq T_{H I}$. For this environment and the instantiation $\pi_{0}$, the set of traces (alternating sequences of locations and actions) of the system is depicted below under the form of an oriented graph, where $q_{i}$, $1 \leq i \leq 9$, are locations of $\mathcal{A}$.

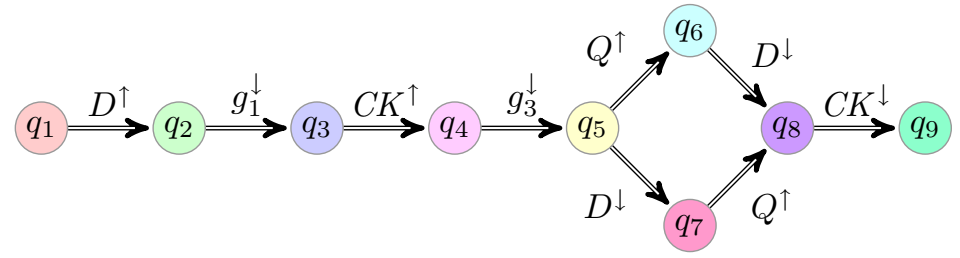

Applying Imitator to $\mathcal{A}$ and $\pi_{0}$, we get the following constraint $K_{0}{ }^{4}$ :

$$
\begin{gathered}
T_{\text {setup }}<T_{L O} \wedge \delta_{3}^{+}+\delta_{4}^{+}<T_{H I} \wedge \delta_{1}^{+}<T_{\text {setup }} \wedge \delta_{1}^{-}>0 \\
\wedge \quad T_{\text {hold }} \leq \delta_{3}^{+}+\delta_{4}^{+} \wedge \delta_{3}^{-}+\delta_{4}^{-} \leq T_{\text {hold }} \wedge \delta_{3}^{+}<T_{\text {hold }}
\end{gathered}
$$

For any valuation $\pi$ satisfying $K_{0}$ and for the same environment, the set of traces of the system $\mathcal{A}[\pi]$ coincides with the one depicted above, i.e., $\mathcal{A}[\pi] \equiv_{T A}$ $\mathcal{A}\left[\pi_{0}\right]$. For a comparison of $K_{0}$ with the constraint found in [7], see [5].

3 The standard parallel composition of several PTAs is a PTA.

${ }^{4}$ It can be surprising that neither $\delta_{2}^{-}$nor $\delta_{2}^{+}$appear in $K_{0}$. This constraint $K_{0}$ actually prevents $G_{2}$ from any change, as $g_{1}$ and $C K$ are never both set to 1 ; therefore, $g_{2}$ always remains set to 1 , and the delay of $G_{2}$ does not have any influence on the system for the considered environment. 


\section{Experiments}

We applied the tool Imitator to various case studies from the literature, including a flip-flop circuit (described in Sect. 4), two protocols (root contention and CSMA/CD), as well as two real case studies: a portion of the memory circuit SPSMALL designed by ST-Microelectronics, and a distributed control system (SIMOP). All those experiments are detailed in [5]. The HyTECH source code of all the examples is available on IMITATOR webpage.

\begin{tabular}{|c||c|c|c|c|c|c||c||c|}
\hline Example & $\begin{array}{c}\text { \# of } \\
\text { PTAs }\end{array}$ & $\begin{array}{c}\text { loc. per } \\
\text { PTA }\end{array}$ & $\begin{array}{c}\text { \# of } \\
\text { clocks }\end{array}$ & $\begin{array}{c}\text { \# of } \\
\text { param. }\end{array}$ & $\begin{array}{c}\text { \# of } \\
\text { iter. }\end{array}$ & $\mid$ Post $^{*} \mid$ & $\left|K_{0}\right|$ & $\begin{array}{c}\text { CPU } \\
\text { time }\end{array}$ \\
\hline Flip-flop [7] & 5 & {$[4,16]$} & 5 & 12 & 8 & 11 & 7 & $2 \mathrm{~s}$ \\
\hline RCP [13] & 5 & {$[6,11]$} & 6 & 5 & 18 & 154 & 2 & $70 \mathrm{~s}$ \\
\hline CSMA/CD [12, 14] & 3 & {$[6,7]$} & 4 & 3 & 21 & 294 & 3 & $108 \mathrm{~s}$ \\
\hline SPSMALL [6] & 10 & {$[3,8]$} & 10 & 22 & 31 & 31 & 23 & $78 \mathrm{mn}$ \\
\hline SIMOP [3] & 5 & {$[6,16]$} & 9 & 16 & 51 & 848 & 7 & $419 \mathrm{mn}$ \\
\hline
\end{tabular}

The above table gives from left to right the name of the example, the number of PTAs composing the system $\mathcal{A}$, the lower and upper bounds on the number of locations per PTA, the numbers of clocks and parameters of $\mathcal{A}$, of iterations of the algorithm, of reached symbolic states, of inequalities in $K_{0}$ (after reduction), and the computation time on an Intel Quad Core $3 \mathrm{GHz}$ with $3.2 \mathrm{~Gb}$.

All these examples are acyclic ${ }^{5}$, and thus guarantee the equality of traces, except SIMOP. In this latter case, we are only interested in avoiding a given bad location, and the equality of reachable locations is sufficient.

In the flip-flop and RCP examples, we took as $\pi_{0}$ an instance satisfying a constraint issued from a classical synthesis method of the literature. In this case, the constraint generated by our method may be the same as the constraint from the literature, but not necessarily : for example, in the case of the flip-flop circuit, $K_{0}$ is uncomparable with the original constraint of [7] (see [5] for details).

In the CSMA/CD, SIMOP, VALMEM examples, the instantiation $\pi_{0}$ corresponds to typical data associated to the case study. In this case, the constraint $K_{0}$ allows us to optimize some values of the typical data $\pi_{0}$. This is useful, for example in order to safely relax some requirements on the environment of asynchronous circuits (see, e.g., [6]). In the SPSMALL case study, this allows us to safely optimize some nominal setup timing by $8 \%$ (see [5]).

Running HYTECH in a brute manner (fully parametric forward analysis) quickly leads to a saturation of the memory for most examples. One reason for which IMiтATOR behaves well in practice is that the procedure drastically reduces the number of reachable states by quickly restraining $K_{0}$.

\section{Final Remarks}

Given a reference valuation $\pi_{0}$, IMITATOR solves the inverse problem for systems modeled by PTA with acyclic traces: it returns a constraint $K_{0}$ on the parame-

\footnotetext{
${ }^{5}$ We considered an acyclic model for CSMA/CD and RCP by bounding the maximal number of collisions of messages.
} 
ters guaranteeing that the sets of traces of $\mathcal{A}\left[\pi_{0}\right]$ and $\mathcal{A}[\pi]$ are identical, for any valuation $\pi$ such that $\pi=K_{0}$.

$K_{0}$ prevents all the bad behaviors (e.g. deadlocks), since it imitates the reference behavior of $\pi_{0}$, while constraints generated by classical methods may not prevent bad behaviors other than those specified by the bad states.

IMITATOR can be used in an incremental way as a complementary tool to enlarge constraints given by classical methods. For example, in the flip-flop case (see Sect. 4), the constraint, say $Z$, found in [7] is uncomparable with our constraint $K_{0}$. We can run Imitator once more with a reference valuation $\pi_{1} \in Z \backslash K_{0}$. This gives a new constraint $K_{1}$, s.t. $K_{0} \cup K_{1}$ is strictly larger than $Z$ (see [5]).

Acknowledgments. I thank anonymous referees for their helpful comments.

\section{References}

1. R. Alur and D. L. Dill. A theory of timed automata. TCS, 126(2):183-235, 1994.

2. R. Alur, T. A. Henzinger, and M. Y. Vardi. Parametric real-time reasoning. In STOC '93, pages 592-601, New York, USA, 1993. ACM.

3. S. Amari, É. André, T. Chatain, O. De Smet, B. Denis, E. Encrenaz, L. Fribourg, and S. Ruel. Timed analysis of distributed control systems combining simulation and parametric model checking. Research report, LSV, ENS Cachan, France, 2009.

4. É. André, T. Chatain, E. Encrenaz, and L. Fribourg. An inverse method for parametric timed automata. International Journal of Foundations of Computer Science (IJFCS). To appear.

5. É. André, E. Encrenaz, and L. Fribourg. Synthesizing parametric constraints on various case studies using IMITATOR. Research report, Laboratoire Spécification et Vérification, ENS Cachan, France, June 2009.

6. R. Chevallier, E. Encrenaz-Tiphène, L. Fribourg, and W. Xu. Verification of the generic architecture of a memory circuit using parametric timed automata. In FORMATS '06, volume 4202 of LNCS, Paris, France, 2006. Springer.

7. R. Clarisó and J. Cortadella. The octahedron abstract domain. In $S A S$ '04, 2004.

8. E. M. Clarke, O. Grumberg, S. Jha, Y. Lu, and H. Veith. Counterexample-guided abstraction refinement. In $C A V$ '00, pages 154-169. Springer-Verlag, 2000.

9. G. Frehse, S.K. Jha, and B.H. Krogh. A counterexample-guided approach to parameter synthesis for linear hybrid automata. In $H S C C$ '08, volume 4981 of $L N C S$, pages 187-200. Springer, 2008.

10. T. A. Henzinger, P. Ho, and H. Wong-Toi. A user guide to HyTech. In TACAS, pages $41-71,1995$.

11. T. Hune, J. Romijn, M. Stoelinga, and F. W. Vaandrager. Linear parametric model checking of timed automata. In TACAS '01, pages 189-203. Springer-Verlag, 2001.

12. X. Nicollin, J. Sifakis, and S. Yovine. Compiling real-time specifications into extended automata. IEEE Trans. on Software Engineering, 18:794-804, 1992.

13. D. Simons and M. Stoelinga. Mechanical verification of the IEEE 1394a Root Contention Protocol using UPPAAL2k. International Journal on Software Tools for Technology Transfer, 3(4):469-485, 2001.

14. Farn Wang. Symbolic parametric safety analysis of linear hybrid systems with BDD-like data-structures. IEEE Trans. Softw. Eng., 31(1):38-51, 2005. 